\title{
Sleep quality is associated with the experience and adaptive regulation of positive
}

emotion: an experience sampling study

Christine E. Parsons ${ }^{1}$, Beatrice Schofield ${ }^{2,3}$, Sofia Batziou ${ }^{4}$, Camilla Ward ${ }^{4}$, Katherine S. Young 4,5

${ }^{1}$ Interacting Minds Center, Department of Clinical Medicine, Aarhus University, Aarhus, Denmark.

${ }^{2}$ Centre for Language Studies, Radboud University, Nijmegen, the Netherlands

${ }^{3}$ Department of Experimental and Neurocognitive Psychology, Freie Universität Berlin, Germany

${ }^{4}$ Social, Genetic and Developmental Psychiatry Centre, Institute of Psychiatry, Psychology and Neuroscience, King's College London, United Kingdom

${ }^{5}$ NIHR Maudsley Biomedical Research Centre, King's College London, UK

Corresponding Author: katherine.s.young@kcl.ac.uk, Phone: (+44) 02078480865

\section{Conflicts of interest}

The authors declare no conflicts of interest.

\section{Acknowledgements}

Christine Parsons received funding from the TrygFonden Charitable Foundation (ID: 117642) and Helsefonden (32062). Katherine Young is supported by funding from the National Institute for Health Research (NIHR) Biomedical Research Centre at South London and Maudsley NHS Foundation Trust and King's College London. The views expressed are those of the author(s) and not necessarily those of the NHS, the NIHR or the Department of Health and Social Care. This work was supported in part by funding from the Psychology and Systems Sciences Divisional Support Fund, King’s College London. 


\begin{abstract}
Poor sleep patterns have been strongly linked to disrupted emotional experiences. Emotion regulation, defined as the capacity to manage one's own emotional responses, is comprised of strategies to increase, maintain, or decrease the intensity, duration, and trajectory of positive and negative emotions. Poor sleep has been identified as a risk factor for emotional dysregulation, but most of the focus has been on negative emotion regulation. We therefore asked whether natural variations in sleep are associated with the experience and regulation of both positive and negative emotion. Young adults, aged between $18-24$ years, $(n=101)$ completed seven-days of ecological momentary assessments using a smartphone application. Duration and quality of the previous night's sleep was reported each morning. Levels of positive and negative emotions, and the strategies used to regulate emotions, were measured at pseudo-random timepoints four times a day. Multilevel modelling indicated that higher self-reported sleep quality was significantly associated with increased intensity and duration of positive emotion, and decreased intensity of negative emotion. Sleep quality, and not sleep duration, was also associated with reported use of adaptive positive emotion regulation strategies. For negative emotion regulation strategy use, we found no associations with sleep quality or duration. Naturally occurring fluctuations in daily sleep quality may be important for the experience and regulation of positive emotion in young adults. These findings emphasise the need to examine both positive and negative emotion, and emotion regulation to understand the links between sleep and mood.
\end{abstract}

Keywords: sleep; emotion regulation; positive emotion; negative emotion; ecological momentary assessment 


\section{Introduction}

Sleep loss and poor sleep quality disrupt how the brain processes emotions (Walker \& van der Helm, 2009). Much of the evidence on the impact of sleep loss on emotion, be it the processing of emotions, the recognition of emotional stimuli, or the capacity to regulate emotion, is derived from studies of full or partial sleep restriction (for review, see Beattie, Kyle, Espie, \& Biello, 2015). Experimentally-reducing sleep to a maximum of 2 hours total has been associated with decreased positive affect, measured using the Positive and Negative Affect Schedule (PANAS), in adolescents and adults (Talbot, McGlinchey, Kaplan, Dahl, \& Harvey, 2010). Even partial sleep restriction, such as reducing sleep by 2 hours per night for 3 nights, has been associated with linear reductions in positive affect across study days (Saksvik-Lehouillier et al., 2020). However, for negative emotion, some studies have reported no change following sleep deprivation in adolescents or adults (measured using the PANAS; Dagys et al., 2012; Saksvik-Lehouillier et al., 2020; Talbot et al., 2010), while others have reported medium-sized adverse effects in adolescents (PANAS; Reddy, Palmer, Jackson, Farris, \& Alfano, 2017).

While experimentally-limiting sleep has disruptive effects on self-reported affect, less is known about the affective experiences associated with natural fluctuations in sleep patterns, particularly in young people. In adult samples (18-61 years), poorer self-reported sleep, both duration and quality, has been associated with reduced positive and increased negative selfreported emotion (de Wild-Hartmann et al., 2013b), and there is some evidence for similar effects in 13 to 16 year olds (van Zundert, van Roekel, Engels, \& Scholte, 2015). However, as noted in a recent systematic review assessing the association between positive affect and sleep, the majority of studies have been cross-sectional, and have methodological challenges 
such as inadequate measurement of negative affect, or small heterogenous sample sizes (Ong, Kim, Young, \& Steptoe, 2017).

\section{What about emotion regulation?}

Another issue in the burgeoning field of emotion-based sleep research is that most studies have focused on the experience of affect that occurs after sleep loss, rather than on the regulatory processes that may alter emotional experiences (Palmer \& Alfano, 2017). Emotion regulation impairments are central to clinical models of anxiety and depression pathogenesis (Clark \& Watson, 1991; Hofmann, Sawyer, Fang, \& Asnaani, 2012), and the role of disrupted negative emotion regulation is especially emphasised. As for studies examining emotion experience, most studies have used experimental reductions in sleep and then assessed negative emotion regulation at a single time point.

One recent study restricting sleep to three hours in young adults found reduced self-reported emotion regulation success, specifically cognitive reappraisal, to negative stimuli (Tamm et al., 2019). In a study of partial sleep reduction to 6.5 hours over 5 nights, Baum et al. (2014) reported emotion reaction difficulties in adolescents, as indicated by self-reported "easily upset" and unprovoked or disproportionate emotional reactions (interpreted as emotion regulation difficulties), relative to after 5 nights of typical sleep. Again, with a focus on negative emotion, an analysis of university students reported that more self-reported sleep difficulties at baseline were associated with reduced self-reported regulation effectiveness one year later (Tavernier \& Willoughby, 2015). However, emotion regulation is not only dependent on the ability to manage responses to the negative. There is now an increasing 
emphasis on the centrality of positive emotion experience, which may be causally disrupted across psychiatric disorders (symptoms of 'anhedonia', Craske et al., 2019).

In the present study, we aimed to address the gaps in knowledge around the role of sleep in positive emotion regulation, while simultaneously recording negative affect and negative emotional regulation. We focused on young adults (aged 18 to 24) because late adolescence and early adulthood is a developmental time point where there are changes in emotion regulation capacities (Young, Sandman, \& Craske, 2019), and striking shifts in sleep patterns (Roenneberg et al., 2004). We used experience sampling to obtain more frequent measurements of young adults' emotional experiences and regulation strategies than afforded by traditional questionnaire measures. Experience sampling also allowed us to measure naturally-occurring fluctuations in participants' sleep in their normal environments. We expected that higher ratings of sleep quality and longer sleep duration would be associated with increased levels of positive affect, and decreased negative affect, consistent with previous studies (de Wild-Hartmann et al., 2013b; Reddy et al., 2017). We also expected that higher ratings of sleep quality and longer sleep would be associated with increased use of adaptive regulation of positive and negative emotions and decreased use of maladaptive strategies, in line with previous studies of negative emotion regulation (Mauss et al., 2013; Palmer et al., 2018). 


\section{Methods}

Figure 1 presents the study design, which consisted of a baseline assessment and a longitudinal EMA component, carried out using the MetricWire (MetricWire Inc.) smartphone application.

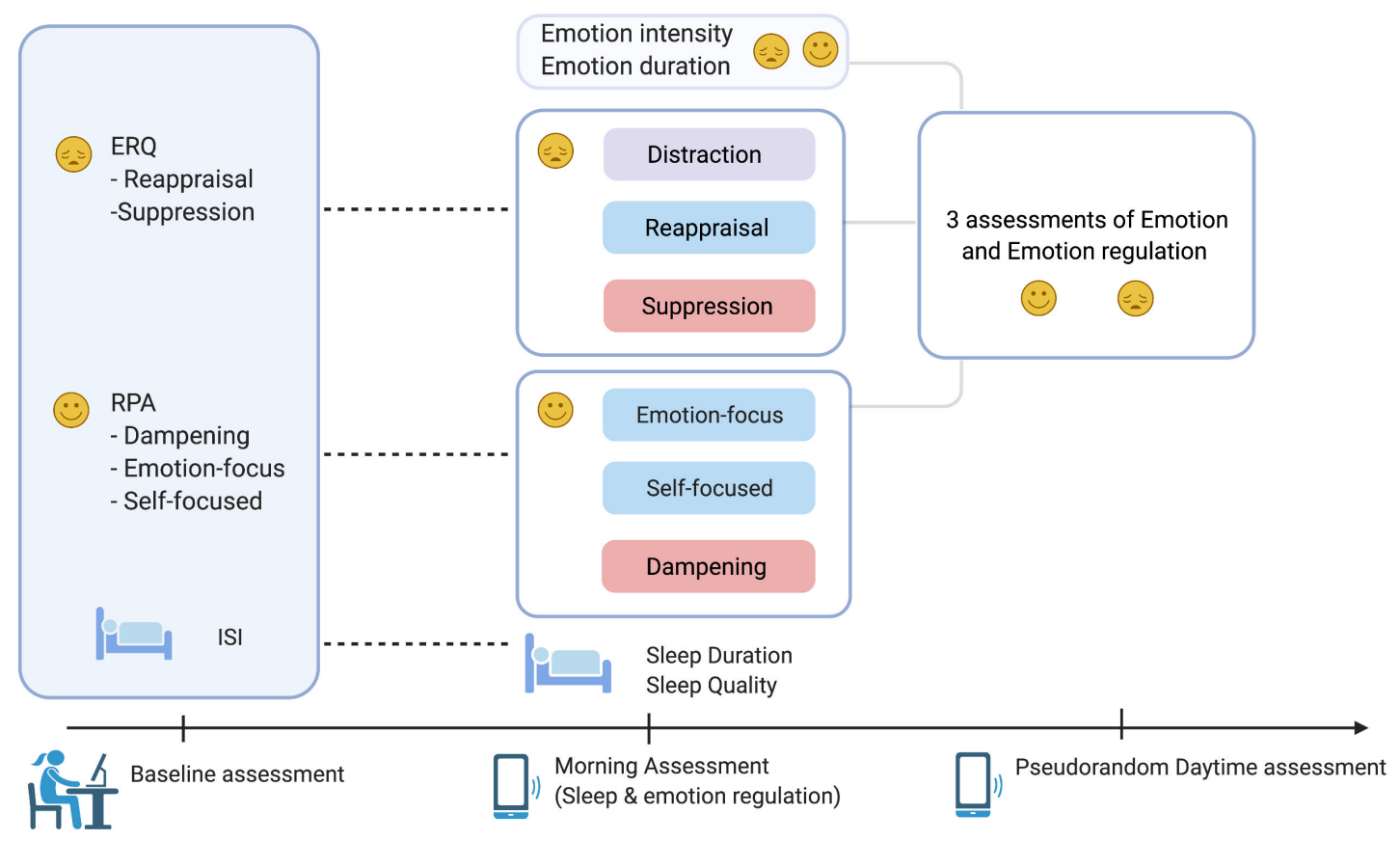

Figure 1. Study design. At baseline, participants completed the Emotion Regulation Questionnaire (ERQ), the Responses to Positive Affect questionnaire (RPA), and the Insomnia Severity Index (ISI). The experience sampling component of the study consisted of daily measures of emotion intensity, emotion duration and emotion regulation strategy use (middle panel). For the emotion regulation strategies (middle panel), adaptive strategies are in blue, maladaptive in red, and neutral in purple. The first survey of each day contained both the sleep assessment and emotion regulation questions, and the other three surveys (final panel) measured emotion levels and regulation only (created using Biorender). 


\section{Participants}

Participants were recruited using a research volunteer system at the Institute of Psychiatry, Psychology and Neuroscience, King's College London, internal departmental webpages, and via social media. Inclusion criteria for participation were: aged between 18-24 years, access to an internet-enabled smartphone device, self-reported as English speaking, and UK residence. No other eligibility criteria were specified. Compensation for participation was comprised of monetary vouchers (£8), with an additional $£ 2$ reward for completion of over $70 \%$ of EMA surveys (67\% of participants completed $>70 \%$ of surveys). Ethical approval for this study was obtained via the Research Ethics Minimal Risk Self-Registration protocol (Daily Emotional Experiences Study, MRA-19/20-19272, Psychiatry, Nursing and Midwifery Research Ethics Subcommittee, King's College London). Participants gave informed consent online via Qualtrics prior to completing the baseline assessment.

We aimed to collect data from at least 100 adults, based on the sample size reported in a previous daily diary study $(\mathrm{N}=98)$, taking measures of emotion intensity and duration over the course of 1 week (Verduyn \& Brans, 2012). One hundred and forty individuals completed the baseline questionnaire, of whom 15 were excluded for not meeting the eligibility criteria (wrong age, $\mathrm{n}=10$; incorrect email, $\mathrm{n}=2$; not resident in the $\mathrm{UK}, \mathrm{n}=3$ ). Of the remaining 125 participants at baseline, 115 individuals registered for the EMA component of the study on the MetricWire smartphone application. Eight of these participants were later excluded because their residence was subsequently reported as outside UK, and six participants provided insufficient data, resulting in a final sample of 101 adults. 


\section{Measures}

At baseline, participants completed a number of measures via Qualtrics (Qualtrics International Inc.), of which we focus on sleep and emotion-related measures for this study (see Table 1 for psychometric properties of each scale). Demographic data was also obtained at baseline (gender, age, and education level, defined as the highest qualification completed).

\section{Sleep}

The Insomnia Severity Index (ISI; Morin, Belleville, Bélanger, \& Ivers, 2011) is a 7-item instrument, widely-used to measure the severity of sleep difficulties and assess the impact these have on an individual's everyday functioning. Participants responded to seven questions using a 5 -point Likert scale ( $0=$ no issues, $4=$ very severe issues $)$.

\section{Emotion regulation}

Positive emotion regulation was assessed using the Responses to Positive Affect questionnaire (RPA; (Feldman et al., 2008), which comprises 6 items evaluating the degree to which participants habitually engage in three different types of positive emotion regulation (2 items for each): (i) adaptive emotion-focused regulation (ii) adaptive self-focused regulation (iii) and maladaptive dampening . Each of these regulation types are reported on a 4-point Likert scale (1=almost never, $4=$ almost always) and as separate subscales within the RPA.

Negative emotion regulation was assessed using the 10-item Emotion Regulation Questionnaire (ERQ, Gross \& John, 2003) which evaluates individual differences in the habitual use of two emotion regulation strategies: (i) adaptive cognitive reappraisal and (ii) maladaptive expressive suppression. Each of these regulation types are reported on a 7-point Likert scale ( $1=$ strongly disagree; 7 strongly agree $)$. 


\section{EMA measures}

Questionnaire items administered during the daily EMA surveys were taken from (i) the Consensus Sleep Diary (Carney et al., 2012), a validated self-report questionnaire designed for daily sleep monitoring, (ii) items assessing positive emotion regulation from the RPA (Feldman et al., 2008) and (iii) items assessing negative emotion regulation, including four items from the ERQ, assessing cognitive reappraisal (2 items) and suppression ( 2 items) and two additional items to measure distraction ("I have been trying to feel less negative by doing something unrelated"; "I have been trying to feel less negative by thinking about something unrelated"), where the first item measured activity-related distraction, derived from Stone et al. (2019), and the second item measured thought-related distraction (see Figure 2). When responding to these questions, participants were asked to "think about your experiences in the last few hours or since the last survey".

\section{Sleep parameters}

Measures of sleep quality and duration were obtained each morning (10am -12pm) with the items (i) 'How would you rate the quality of your sleep?' (5-point Likert scale, 1 = very poor, 5 = very good) and (ii) 'What time did you go to sleep?' and 'What time did you wake up?', with duration calculated to the nearest half hour from the participant-estimated sleep and wake times.

\section{Emotion and emotion regulation}

Positive and negative emotion intensity were assessed using the two ratings: 'How positive have you been feeling?' and 'How negative have you been feeling?' on a sliding scale between 0-100. Positive and negative emotion duration were assessed using the item "How 
long has this feeling been going on?", on a sliding scale ranging from 'a few seconds' to 'a few hours', which was then converted to a score between 0 to 100 . The use of regulation strategies was then assessed using the question 'What types of thoughts have you been having about these feelings?' for each emotion type. Participants were able to select as many strategies as applicable (see Figure 2).

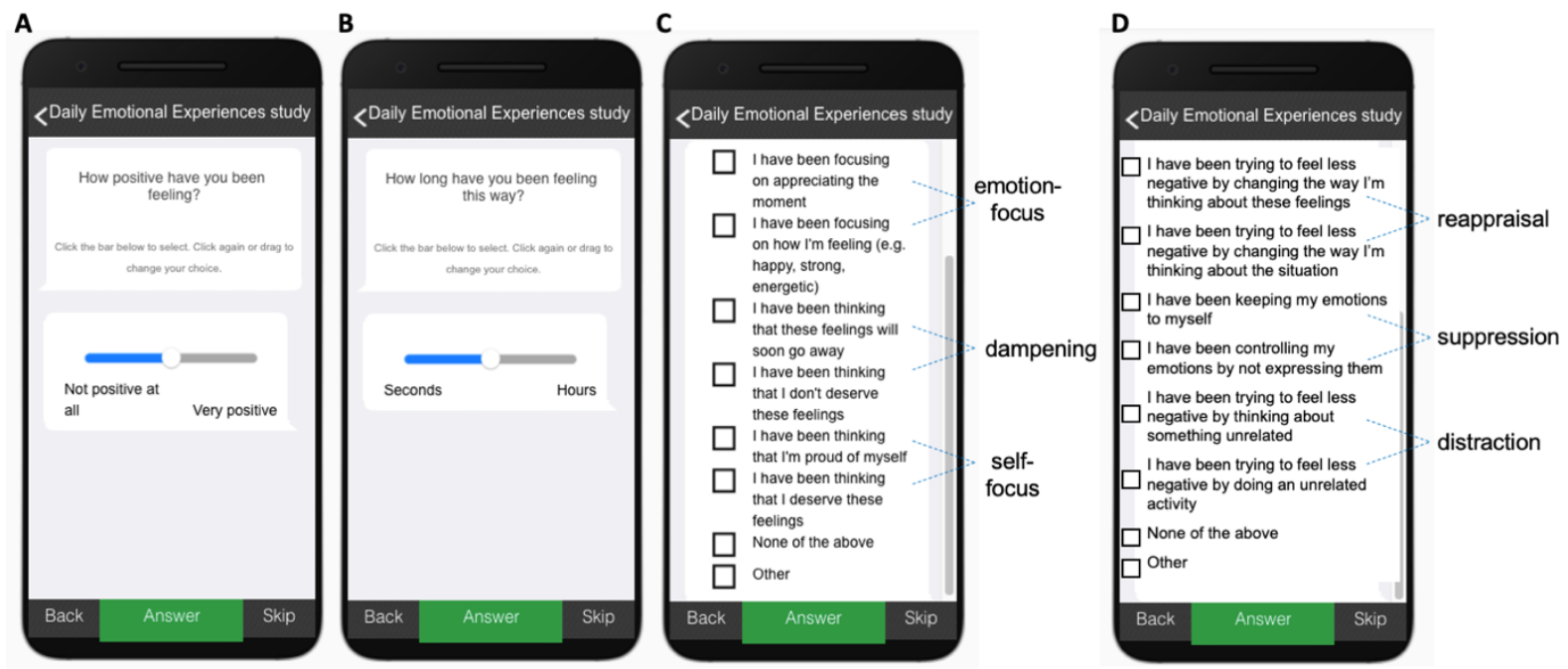

Figure 2: Smartphone EMA measurement: A. Assessing emotion intensity using a sliding visual analogue scale (VAS; prompts asked 'how positive' or 'how negative'). B. Assesing emotion duration using a sliding VAS. C-D. Assessing strategies used for regulating emotions using a tick box format, for both postive (C) and negative (D) emotion regulation strategies.

\section{Procedure}

After completion of the initial baseline measures, participants were contacted by a researcher by email and familiarised with the MetricWire smartphone application and the EMA procedures. After registration within the MetricWire application, participants received four questionnaires each day for seven days (28 questionnaires in total, see Figure 1). 
A notification for the first survey of the day was sent to participants at a random timepoint between 10 am and $12 \mathrm{pm}$, while notifications for the following three surveys were sent at pseudo-random times (at least two hours apart) between $1 \mathrm{pm}$ and $10 \mathrm{pm}$. Links to surveys expired after 20 minutes and were recorded as missed if not returned within this period. A threshold of $20 \%$ of completed surveys was required in order for data to be incorporated into subsequent analyses, as per recommendations for EMA procedures to ensure sufficient power (Edwards, Cella, Tarrier, \& Wykes, 2016). Finally, to encourage engagement over the duration of the experiment, participants whose responses dropped below $40 \%$ by the third day of surveys were contacted by email to remind them about the participation bonus.

\section{Statistical analysis}

Data processing and analysis was carried out in RStudio version 4.0.2 (The R Development Core Team, 2020). Data collected within the EMA portion of the study was nested in three levels: observations (i.e. surveys), within days (note sleep parameters were measured just once per day), within participants. Multilevel modelling was used because it can accommodate the hierarchical structure of EMA data (Carter, 2016). All R code is available from: https://osf.io/urjsf/.

For each of the outcomes (EMA measures of emotion intensity, emotion duration, and emotion regulation strategies), three-level, random-intercept models were created, with each of the day-level sleep parameters (sleep duration, sleep quality) added as predictors. We used baseline participant-level ISI scores, RPA scores, and ERQ scores as covariates. 'Participant' and 'Days within participant' were added as random intercepts to account for between-person 
and between-day differences respectively throughout these analyses, as per previous threelevel EMA procedures (Geyer et al., 2018). For multilevel models, we used the lme4 package within $\mathrm{R}$, and an optimization by quadratic approximation (BOBYQA) with a set maximum of 20,000 iterations.

We first tested whether the daily EMA measures of sleep (duration and quality) were associated with emotion intensity (positive, negative; 0 -100 scale) and emotion duration (positive, negative; 0 -100 scale). Next, we tested whether sleep (duration, quality) was associated with emotion regulation (use of strategies coded as binary variables: $0=$ not used, $1=$ used). A binarized measure of use of each regulation strategy type at each survey timepoint per participant was created.

Unlike models with continuous outcomes, which represent expected effects at both the participant and sample level, the results of multilevel models with binary outcome variables are participant-specific only (Hox, 2010). Therefore, sample-level trends were calculated in the form of predicted probabilities as per recommendations for logistic models (Persoskie \& Ferrer, 2017). All analyses were conducted using maximum likelihood estimates (McCulloch, 2003). All predictors were centered, with time-varying predictors centered using the individual's mean and time-invariant predictors using the grand mean (Snijders \& Bosker, 2012). Sleep duration was assessed as a linear predictor in all models, consistent with previous EMA designs (Littlewood et al., 2019; Short, Allan, \& Schmidt, 2017).

Outliers were calculated by generating weighted averages (using the number of surveys returned by each participant returned) for each variable. Outliers were defined as where the participant's average exceeded IQR +1.5 or fell below IQR -1.5. We repeated analyses with 
and without outliers and found similar patterns of significant effects (see Supplementary materials for the analyses presented with and without outliers).

\section{Results}

One hundred and one individuals ( 85 female, 16 male) took part in this study, with a mean age of 21.69 years $(\mathrm{SD}=1.91$; range $18-24$ years). Approximately half had completed secondary school level education (45.54\%), and half had obtained higher education qualifications (bachelor's degree: $37.62 \%$, master's degree: 14.85\%). Descriptive statistics for baseline assessments of sleep and trait-level emotion regulation tendencies are shown in Table 1.

Table 1. Descriptive statistics for baseline measures of sleep and trait-level emotion regulation $(\mathrm{N}=101)$

\begin{tabular}{lclll}
\hline & Mean (SD) & Scale range & Sample range & Cronbach's $\alpha$ \\
\hline ISI & $9.40(5.46)$ & $0-28$ & $0-24$ & .86 \\
RPA: emotion focused & $12.65(2.85)$ & $5-20$ & $5-20$ & .73 \\
RPA: self-focused & $8.22(2.69)$ & $4-16$ & $4-16$ & .80 \\
RPA: dampening & $18.61(4.93)$ & $8-32$ & $9-29$ & .82 \\
ERQ: cognitive reappraisal & $27.40(6.31)$ & $6-42$ & $11-38$ & .84 \\
ERQ: expressive suppression & $14.75(5.62)$ & $4-28$ & $4-25$ & .84 \\
\hline
\end{tabular}

Note: ISI $=$ insomnia severity index; RPA $=$ responses to positive affect scale; ERQ = emotion regulation questionnaire

Mean experience sampling survey completion rate was $72.77 \%(\mathrm{SD}=17.53)$. From the experience sampling measures, the mean sleep quality score was 3.40 (averaged across days, 
then across individuals; range $1.5-5, \mathrm{SD}=.68)$ and mean sleep duration was 8.01 hours (SD $=.99)$. Mean emotion and regulation ratings are displayed in Figure 3.

For positive regulation strategies, the most frequently used was emotion-focused strategies (58.11\% of all reports). Self-focused emotion regulation (19.28\%) and dampening (11.46\%) were less frequently used. For negative emotion regulation, the frequency of reported use was similar for all three regulation strategies (reappraisal: $29.28 \%$, suppression: $23.46 \%$, distraction: $38.72 \%$ ). Further descriptive statistics for the experience sampling emotion regulation ratings are presented in Table 2. Within- and between-person standard deviations indicate that strategy use varied at least as much within individuals as between individuals. Intra-class correlations show that $12 \%-47 \%$ of the variance within each individual strategy was accounted for by between-person variation.

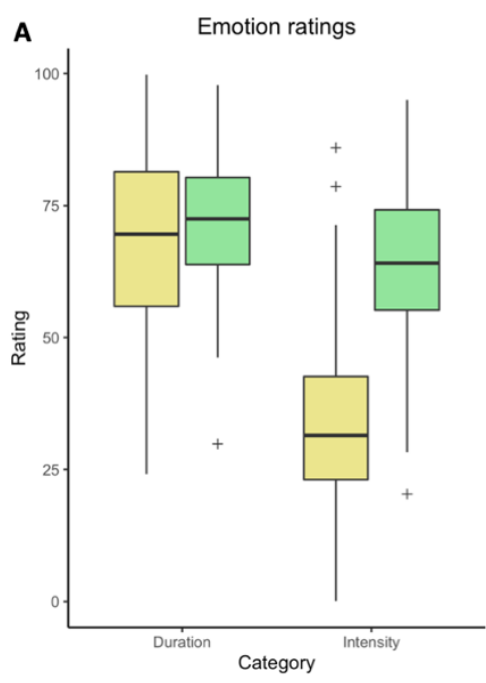

Emotion 官 negative 官 positive

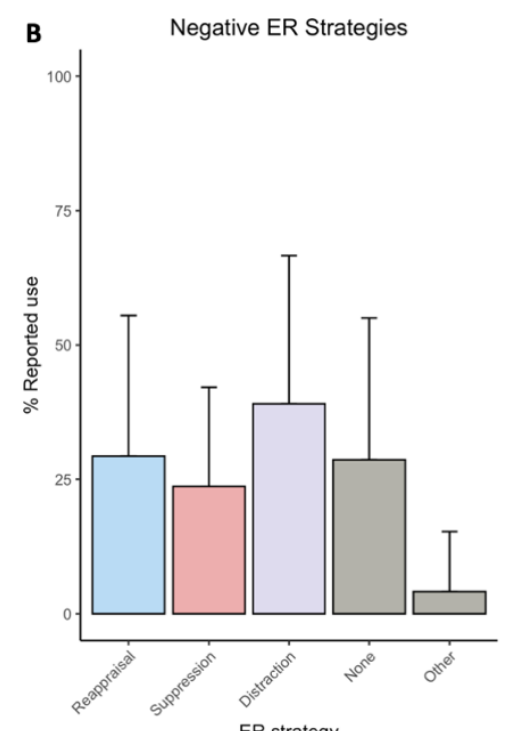

ER strategy

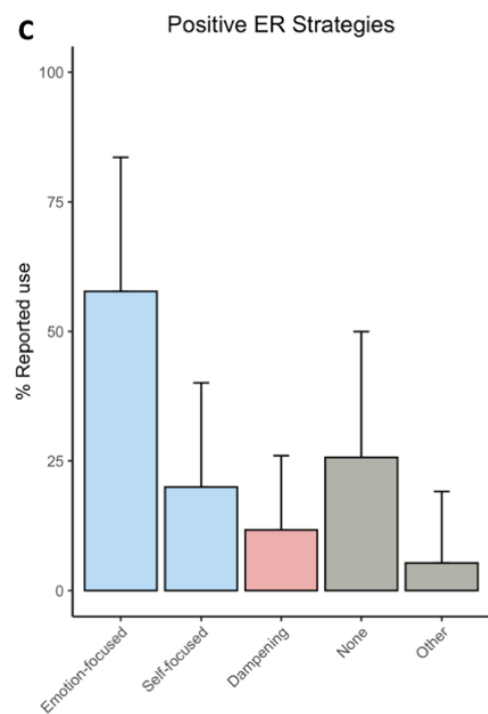

ER strategy

Figure 3. Mean ratings of reported emotion intensity and duration (A) and regulation strategy use (B and C). Error bars indicate mean +/- standard deviation, '+' in panel A denotes outlier values. Note that individuals were able to select more than one regulation strategy at each EMA prompt (therefore total across strategy usage $>100 \%$ ). 
Table 2. Descriptive statistics for the experience sampling Emotion Regulation (ER) strategies. ICC: intra-class correlations at the subject level, denoting the amount of variance attributable to between-person effects

\begin{tabular}{|c|c|c|c|c|c|c|c|}
\hline ER strategy & Item & $M^{*}$ & $\begin{array}{l}95 \% \mathrm{CI} \\
\text { lower }\end{array}$ & $\begin{array}{l}95 \% \text { CI } \\
\text { upper }\end{array}$ & $\begin{array}{l}\text { SD between- } \\
\text { person }\end{array}$ & $\begin{array}{l}\text { SD within- } \\
\text { person residual }\end{array}$ & ICC \\
\hline \multicolumn{8}{|l|}{ Emotion- } \\
\hline & 1 & 0.43 & 0.38 & 0.48 & 0.25 & 0.29 & 0.42 \\
\hline & 2 & 0.33 & 0.28 & 0.38 & 0.22 & 0.28 & 0.38 \\
\hline \multirow[t]{2}{*}{ Self-focus } & 1 & 0.14 & 0.10 & 0.17 & 0.15 & 0.21 & 0.34 \\
\hline & 2 & 0.11 & 0.08 & 0.14 & 0.13 & 0.18 & 0.32 \\
\hline \multirow[t]{2}{*}{ Dampening } & 1 & 0.08 & 0.06 & 0.10 & 0.06 & 0.17 & 0.12 \\
\hline & 2 & 0.05 & 0.03 & 0.07 & 0.08 & 0.13 & 0.29 \\
\hline \multirow[t]{2}{*}{ Reappraisal } & 1 & 0.16 & 0.12 & 0.20 & 0.18 & 0.22 & 0.40 \\
\hline & 2 & 0.21 & 0.16 & 0.26 & 0.22 & 0.23 & 0.47 \\
\hline \multirow[t]{2}{*}{ Suppression } & 1 & 0.18 & 0.15 & 0.21 & 0.14 & 0.24 & 0.25 \\
\hline & 2 & 0.12 & 0.10 & 0.15 & 0.10 & 0.21 & 0.20 \\
\hline \multirow[t]{2}{*}{ Distraction } & 1 & 0.20 & 0.16 & 0.24 & 0.19 & 0.23 & 0.41 \\
\hline & 2 & 0.32 & 0.27 & 0.37 & 0.25 & 0.27 & 0.47 \\
\hline
\end{tabular}

*mean proportion of strategy use per day, averaged across participants

How are baseline trait measures and EMA measures related?

Bivariate correlations between baseline measures of sleep and emotion regulation and EMA measures of sleep, emotion, and emotion regulation are presented in Figure 4. Among sleep measures, baseline insomnia severity was significantly associated with EMA-measured mean sleep quality, but not duration, and mean quality and duration were significantly associated 
with each other. For EMA emotion measures, positive emotion intensity and duration were significantly correlated, with a medium effect size $(r=.55)$, but negative emotion and intensity were not significantly correlated $(r=-.18)$. Across baseline trait emotion regulation subscales: i) self-focused and emotion-focused positive emotion regulation were significantly positively correlated, ii) neither of these subscales were significantly correlated with dampening positive emotion regulation, iii) negative emotion suppression and reappraisal were not significantly correlated. Similar patterns were observed among EMA state emotion regulation subscales, with the addition that both state level suppression and reappraisal were significantly associated with distraction.

To examine the validity of state (EMA) emotion regulation measures, we first examined the association between state and trait-level baseline measures of the same regulation strategy. For positive emotion, EMA measures of emotion-focused, and self-focused regulation (adaptive strategies) were significantly correlated with trait measures of the same subscales, but not with a trait measure of dampening. EMA dampening was significantly associated only with trait level dampening (maladaptive strategy). For negative emotion, EMA reappraisal was significantly associated only with trait level reappraisal and EMA suppression was significantly associated only with trait level suppression. EMA distraction was not significantly associated with trait level reappraisal or suppression.

Next, we examined the relationship between EMA emotion regulation strategies and EMA emotion intensity and duration (Figure 4). For positive emotions, greater use of emotionfocused or self-focused regulation and less use of dampening was associated with more intense positive emotions. Higher levels of dampening were associated with shorter emotion duration. For negative emotions, greater EMA reported use of suppression and distraction 
was significantly associated with more intense negative emotions. Greater reported use of suppression was also associated with shorter negative emotion duration. [Note that bivariate correlations of measures between categories (i.e., between sleep and emotion regulation) are presented for completeness, but findings are described in the results of multilevel model sections, as these analyses more accurately represent the nested data structure.]

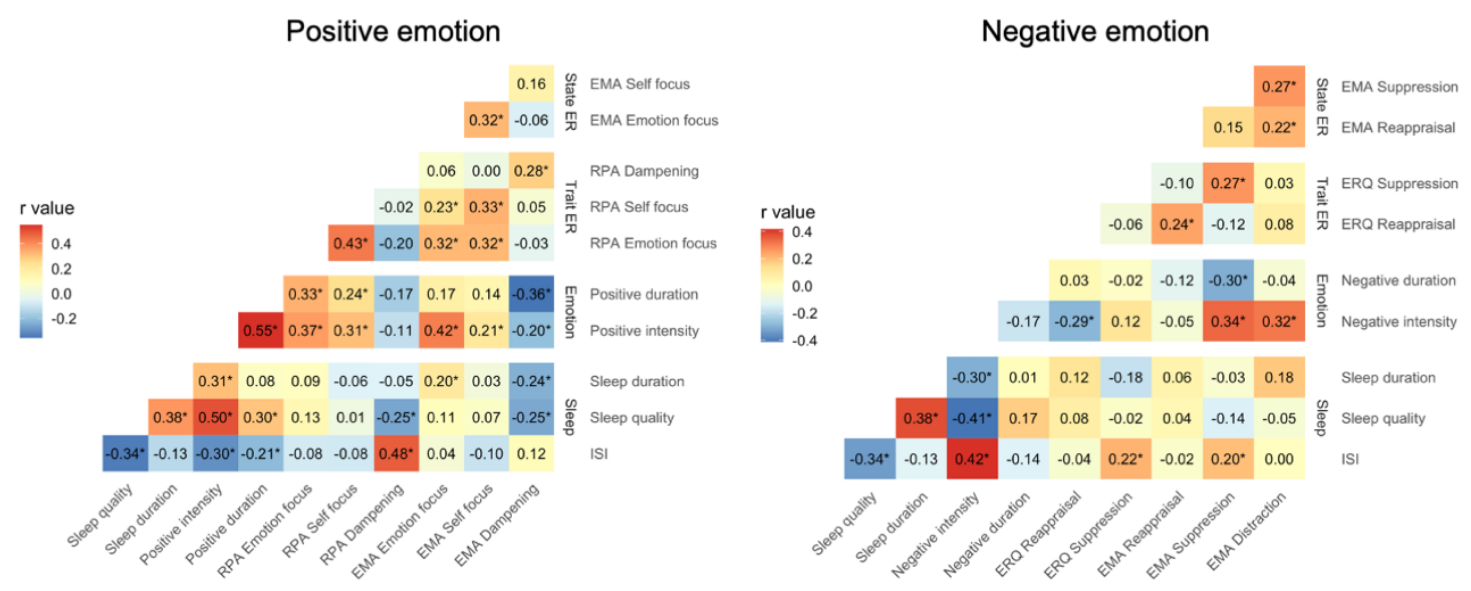

Figure 4. Heatmaps of bivariate correlations between sleep (ISI and EMA-reported Sleep quality, Sleep duration), emotion (EMA-reported intensity, duration) and state (EMA) and trait emotion regulation measures (RPA, ERQ) presented separately for positive and negative emotion and regulation strategies $(\mathrm{N}=101)$. [* denotes significance at $p<.05$; EMA: ecological momentary assessment, ER: emotion regulation, ERQ: emotion regulation questionnaire, ISI: insomnia severity index, RPA: responses to positive affect scale]

\section{Effects of sleep on positive and negative emotion intensity and duration}

Four multilevel models examined the effect of daily variation in self-reported sleep quality and duration on daily levels of: i) positive emotion intensity, ii) positive emotion duration, iii) negative emotion intensity, and iv) negative emotion duration. 


\section{Positive emotion: experience and duration}

For positive emotion intensity, there were significant effects of both daily sleep quality and daily sleep duration. Higher quality and longer sleep were associated with higher daily ratings of positive emotion intensity (sleep quality: $\beta=5.87, p<.001$; sleep duration: $\beta=$ 2.31, $p=.01$, Table S1; Table S3 for results of models including outliers). In this model, the ISI and two subscales of the RPA (emotion-focused and self-focused positive emotion regulation) were significant: lower levels of insomnia symptoms and greater trait use of both emotion-focused and self-focused regulation were associated with higher positive emotion intensity (ISI: $\beta=-.62, p=.01$; RPA emotion-focused: $\beta=1.02, p=.03$; RPA self-focused: $\beta=1.14, p=.02)$. There was no significant effect of the RPA dampening subscale $(\beta=.22, p$ $=.44)$.

For positive emotion duration, higher ratings of daily sleep quality were associated with longer duration of positive emotions $(\beta=2.30, p=.03)$, but ratings of daily sleep duration were not significant $(\beta=.80, p=.31)$. In this model, greater use of emotion-focused regulation was associated with longer positive emotion duration $(\beta=1.18, p=.03)$. The ISI and other two RPA subscales were not significant (ISI: $\beta=-.46, p=.10$; RPA self-focused: $\beta$ $=.54, p=.34$; RPA dampening: $\beta=-.14, p=.67$ ).

\section{Negative emotion: experience and duration}

For negative emotion intensity, there were significant effects of both daily sleep quality and daily sleep duration. Lower quality and shorter sleep were associated with higher ratings of negative emotion intensity (sleep quality: $\beta=-5.66, p<.001$; sleep duration: $\beta=-2.58, p=$ .01; Table S2; Table S4 for results of models including outliers). In this model, the ISI and 
the ERQ cognitive reappraisal subscale were significant: higher levels of insomnia symptoms and less trait-level use of cognitive reappraisal were associated with higher negative emotion intensity (ISI: $\beta=.92, p<.001$; ERQ cognitive reappraisal: $\beta=-.44, p=.04$ ). The ERQ expressive suppression subscale was not significant $(\beta=.08, p=.74)$.

For negative emotion duration, there were no significant effects of daily sleep quality, or duration (sleep quality: $\beta=.28, p=.82$; sleep duration: $\beta=-.39, p=.68$ ). In this model, the ISI and ERQ subscales were also not significantly associated with negative emotion duration (ISI: $\beta=-.54, p=.10$; ERQ cognitive reappraisal: $\beta=.11, p=.70$; ERQ expressive suppression: $\beta=.14, p=.65)$.

\section{Effects of sleep on emotion regulation strategy use}

Six multilevel models examined the effect of daily variation in sleep quality and duration on daily levels of emotion regulation strategy use (3 positive regulation strategies, 3 negative regulation strategies).

\section{Positive emotion regulation strategies}

For emotion-focused regulation, higher daily sleep quality was associated with greater daily use of emotion-focused regulation $(\beta=.31, p=.03$; Figure 5 , Table S1; Table S3 for results of models including outliers). There were no significant effects of sleep duration $(\beta=.14, p=$ .20). In this model, higher trait level emotion-focused regulation was significantly associated with more frequent daily emotion-focused regulation $(\beta=.19, p=<.001)$. There were no significant effects of insomnia symptoms, or the other subscales of the RPA (ISI: $\beta=.01, p=$ .85 ; RPA self-focused: $\beta=.06, p=.40$; RPA dampening: $\beta=.06, p=.11$ ). 

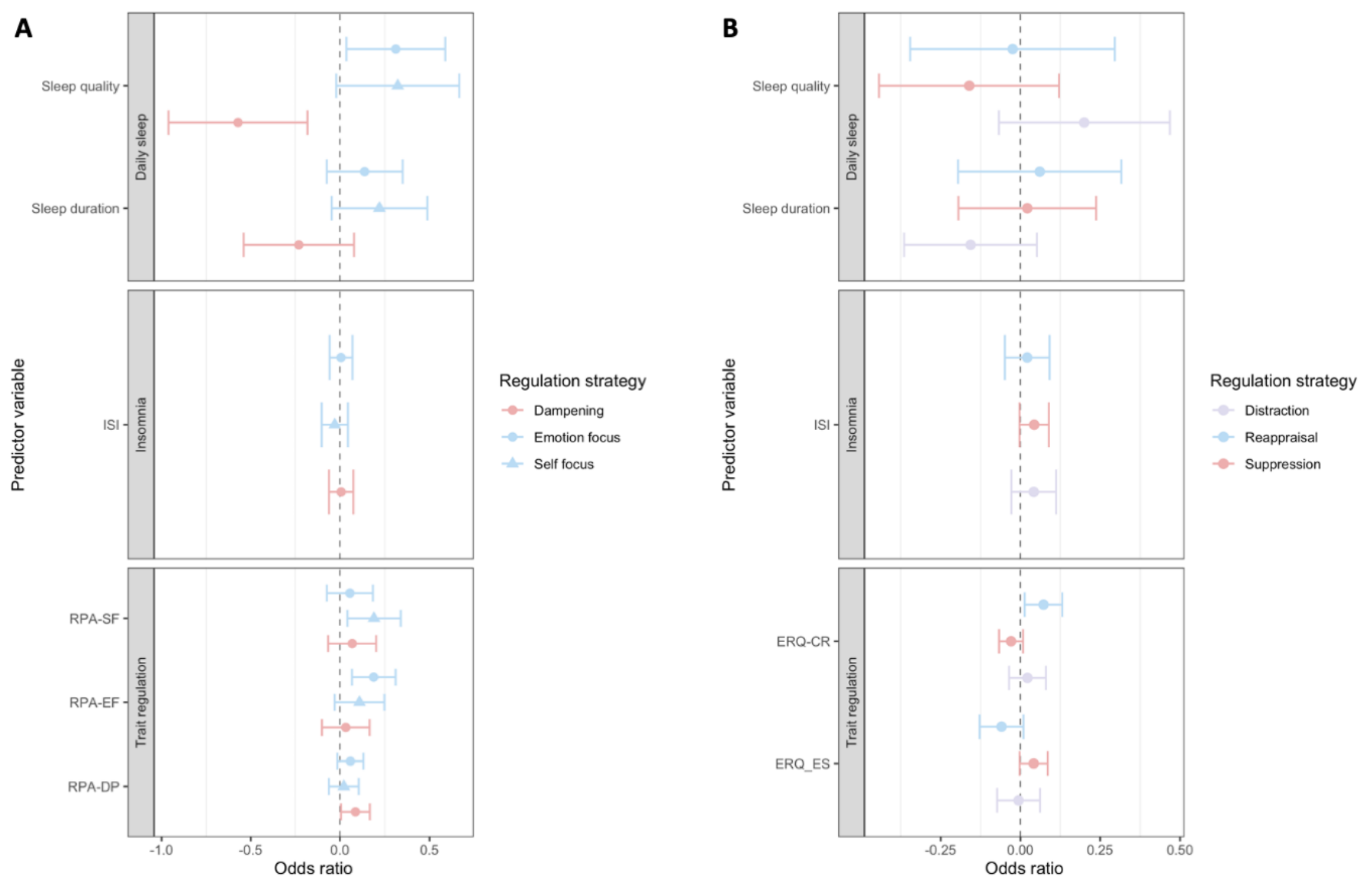

Figure 5. Results of multilevel regression models with sleep (daily ESM-reported sleep, baseline ISI) and trait-level emotion regulation (RPA; ERQ) as predictors of daily emotion regulation strategy use. A) For positive emotion regulation strategies, higher daily sleep quality was associated with significantly more 'emotion focus', and significantly less 'dampening' strategy use. B) For negative emotion regulation strategies, there were no significant associations with daily sleep quality or duration.

For self-focused regulation, there were no significant effects of daily sleep quality or duration (sleep quality: $\beta=.32, p=.07$; sleep duration: $\beta=.22, p=.11$ ). In this model, higher traitlevel self-focused regulation was significantly associated with more frequent daily selffocused regulation $(\beta=.19, p=.01)$. There were no significant effects of insomnia symptoms 
or the other subscales of the RPA (ISI: $\beta=-.03, p=.44$; RPA emotion-focused: $\beta=.11, p=$ .12; RPA dampening: $\beta=.02, p=.61)$.

For dampening regulation, higher daily sleep quality was significantly associated with less frequent daily use of dampening regulation $(\beta=-.57, p=.004)$. There was no significant effect of sleep duration $(\beta=-.23, p=.14)$. In this model, higher trait-level dampening was significantly associated with more frequent daily use of dampening $(\beta=.09, p=.04)$. There were no significant effects of insomnia symptoms or the other subscales of the RPA (ISI: $\beta=$ $.01, p=.84$; RPA emotion-focused: $\beta=.03, p=.63$; RPA self-focused: $\beta=.07, p=.32$ ). In sum, for positive emotion regulation, we found that sleep quality was associated with more use of an adaptive emotion regulation strategy (emotion-focus), and less use of maladaptive strategy (dampening).

\section{Negative emotion regulation strategies}

For cognitive reappraisal, there were no significant effects of daily sleep quality or duration (sleep quality: $\beta=-.02, p=.88$; sleep duration: $\beta=.06, p=.64$; Figure 5, Table S2; Table S4 for results of models including outliers). In this model, greater trait-level use of cognitive reappraisal was significantly associated with greater daily use of cognitive reappraisal $(\beta=$ $.07, p=.02)$. There were no significant effects of insomnia symptoms, or the other subscale of the ERQ (ISI: $\beta=.02, p=.55$; ERQ expressive suppression: $\beta=-.06, p=.09$ ).

For expressive suppression, there were no significant effects of daily sleep quality or duration (sleep quality: $\beta=-.16, p=.26$; sleep duration: $\beta=.02, p=.85$ ). There were also no significant effects of insomnia symptoms or either subscale of the ERQ on daily levels of expressive suppression (ISI: $\beta=.04, p=.07$; ERQ cognitive reappraisal: $\beta=-.03, p=.12$; 
ERQ expressive suppression: $\beta=.04, p=.07)$. In the model including outliers, there was a significant relationship between trait-level use of expressive suppression and daily use of expressive suppression (see Table S4), but otherwise there were no differences between the models including or excluding outliers.

For distraction, there were no significant effects of daily sleep quality or duration (sleep quality: $\beta=.20, p=.14$; sleep duration: $\beta=-.16, p=.14$ ). There were also no significant effects of insomnia symptoms or either subscale of the ERQ on daily levels of distraction use (ISI: $\beta=.04, p=.25$; ERQ cognitive reappraisal: $\beta=.02, p=.46$; ERQ expressive suppression: $\beta=-.01, p=.86$ ). In sum, in contrast to that seen for positive emotion, there were no significant associations between sleep quality and emotion regulation strategy use for negative emotion.

\section{Discussion}

Using experience sampling in young adults, we found that naturally-occurring variations in at home-sleep patterns were associated with the daily experience of positive emotion, both its intensity and duration. Sleep quality, in particular, was associated not just with the experience of positive emotion, but also with reported engagement in strategies to regulate it. Young adults with higher ratings of quality sleep tended to report greater engagement in an adaptive regulation strategy, taking an emotion-focus approach (e.g., appreciating the moment), and less engagement in a maladaptive regulation strategy, dampening (e.g., thinking the positive feelings will go away). Longer sleep duration was associated only with intensity of daily positive emotion experience, and not its duration, or with engagement in any of the tested regulation strategies. 
For negative emotion, and negative emotion regulation, sleep pattern associations were apparent only for the measure of emotion intensity. Both higher sleep quality and longer sleep duration were associated with reporting of less intense daily negative emotion. We examined participants' reports of three negative emotion regulation strategies, cognitive reappraisal, suppression, and distraction, and none of these were significantly associated with sleep quality or duration.

While a large body of studies have illustrated links between sleep and general mood, or sleep and PANAS-measured affect (Ben Simon, Vallat, Barnes, \& Walker, 2020), we show that sleep is also associated with reported use of positive emotion regulation strategies. We measured young adults reported daily tendencies to engage in regulation strategies that can influence the experience of positive emotion, multiple times per day over the course of a week, controlling for trait regulation abilities measured at baseline (ERQ, RPA) and sleep difficulties (ISI). Our focus on emotion regulation is of importance, because poor regulation is a transdiagnostic risk factor for psychopathology (Aldao, Nolen-Hoeksema, \& Schweizer, 2010), and effective regulation of positive emotion is associated with life satisfaction (Quoidbach, Berry, Hansenne, \& Mikolajczak, 2010).

As has been recommended (Palmer \& Alfano, 2017), we measured both positive and negative emotion experiences, as well as a range of well-defined emotion regulation strategies. Moving beyond cross-sectional measurements of a single regulation strategy, our use of EMA allowed us to capture day-to-day variations in strategy use in a sample of young adults. For positive emotion, we tested two adaptive positive regulation strategies: emotion-focus and self-focus (e.g., taking pride), and one maladaptive strategy (dampening). Self-focus was not significantly associated with sleep quality, whereas the effects for the other two strategies 
were small to moderate. We found no effects for negative emotion regulation strategies, in contrast to work restricting sleep in young adults, which reported reduced cognitive reappraisal success after less sleep (Tamm et al., 2019). We speculate that, because our participants broadly fell within the healthy 7-9-hour sleep range, their sleep may have been insufficiently variable to detect effects that measurable in a more severe experimental condition of just 3 hours sleep.

\section{Comparing trait and daily measures of emotion regulation}

We obtained baseline indices of emotion regulation, using two well-established questionnaire instruments the ERQ and the RPA, with reasonable psychometric properties (test-retest reliability for RPA subscales from 0.51-0.65; for both ERQ subscales: 0.69, internal consistency for RPA subscales from $0.62-0.8$, and for the ERQ from 0.73 to 0.79 (Gross \& John, 2003; Raes, Smets, Nelis, \& Schoofs, 2012)). We found that participants' responses on the ERQ and RPA subscales were significantly correlated with the related EMA measures: for example, trait “suppression" (ERQ) correlated with EMA-measured suppression. Effect sizes were in the small to medium range (Fig. 4), as might be anticipated for trait-to-daily measure correlations. We interpret these correlations as indicating that our EMA measures are reasonably related to the constructs measured in the two standardised questionnaire instruments.

\section{Limitations and future directions}

Our study was observational and complementary to the controlled experimental studies that have directly manipulated sleep (e.g., Baum et al., 2014; Tamm et al., 2019). While we believe that the assessment of natural fluctations in sleep are of interest, we cannot speak to the directionality of effects with the current design and analyses. Relatedly, we treated sleep duration as a linear variable, consistent with previous EMA studies (Littlewood et al., 2019; 
Short et al., 2017). However, both under-sleeping (less than 5 hours) and oversleeping (e.g., over 9 hours) have been associated with health outcome impairments (Itani, Jike, Watanabe, \& Kaneita, 2017; Jike, Itani, Watanabe, Buysse, \& Kaneita, 2018). Future work may look to sample participants falling outside of the 7-9-hour range typically characterised as healthy, and may include objective measurements of actual sleep time and more detailed analyses of chronotype and sleep pattern regularity.

Our participants were asked at each EMA prompt to record current emotions, and how they were regulating these emotions, but were not required to describe the context of emotional experiences themselves. The appropriateness of a regulation strategy is likely to differ depending on the context, so consideration of environmental conditions will be important in future work. To give an example, although dampening is generally defined as maladaptive to positive emotions, if an individual was in a serious situation at the time of the survey prompt, reducing the expression of their positive emotions may have been socially appropriate or culturally expected (John \& Gross, 2004). Our measure of regulation strategy use can be characterised as an assessment of participants' tendencies, or reported use of strategies, as distinct from an assessment of their emotion regulation ability (e.g., using measures of efficacy of a strategy to upregulate or downregulate an emotion, see for example Reddy et al. (2017)). While our assessments of trait-level emotion regulation at baseline were comprised of questionnaires, it would also be possible to combine laboratory measures of regulation with EMA methodology in future work.

\section{Conclusion}

Positive and negative emotion intensity were associated with sleep duration and quality, as predicted from previous studies (Palmer \& Alfano, 2017). Sleep quality, but not sleep 
duration, was associated with daily adaptive use of positive emotion regulation strategies, as measured in young adults using EMA. These findings add to the body of studies emphasising the importance of sleep quality over sleep duration in emotion experience (e.g., Shen, van Schie, Ditchburn, Brook, \& Bei, 2018). Our findings emphasise the link between sleep and positive emotion, and are broadly consistent with studies suggesting that poor sleep quality is more robustly linked to impaired positive relative to negative mood (e.g., Bower, Bylsma, Morris, \& Rottenberg, 2010; de Wild-Hartmann et al., 2013a). We suggest that emotion regulation strategy use may be a candidate mechanism linking poor sleep quality and disrupted positive mood, which could be investigated further in experimental designs. 
Running head: Sleep quality and positive emotion regulation

\section{References}

Aldao, A., Nolen-Hoeksema, S., \& Schweizer, S. (2010). Emotion-regulation strategies across psychopathology: A meta-analytic review. Clinical Psychology Review, 30(2), 217-237. doi:https://doi.org/10.1016/j.cpr.2009.11.004

Baum, K. T., Desai, A., Field, J., Miller, L. E., Rausch, J., \& Beebe, D. W. (2014). Sleep restriction worsens mood and emotion regulation in adolescents. $J$ Child Psychol Psychiatry, 55(2), 180-190. doi:10.1111/jcpp.12125

Beattie, L., Kyle, S. D., Espie, C. A., \& Biello, S. M. (2015). Social interactions, emotion and sleep: A systematic review and research agenda. Sleep Medicine Reviews, 24, 83-100. doi:https://doi.org/10.1016/j.smrv.2014.12.005

Ben Simon, E., Vallat, R., Barnes, C. M., \& Walker, M. P. (2020). Sleep Loss and the SocioEmotional Brain. Trends in Cognitive Sciences, 24(6), 435-450. doi:10.1016/j.tics.2020.02.003

Bower, B., Bylsma, L. M., Morris, B. H., \& Rottenberg, J. (2010). Poor reported sleep quality predicts low positive affect in daily life among healthy and mood-disordered persons. Journal of Sleep Research, 19(2), 323-332.

Carney, C. E., Buysse, D. J., Ancoli-Israel, S., Edinger, J. D., Krystal, A. D., Lichstein, K. L., \& Morin, C. M. (2012). The consensus sleep diary: standardizing prospective sleep self-monitoring. Sleep, 35(2), 287-302. doi:10.5665/sleep.1642

Carter, L. A. (2016). Rigorous methods for the analysis, reporting and evaluation of ESM style data. . (PhD). The University of Manchester, UK.

Clark, L. A., \& Watson, D. (1991). Tripartite model of anxiety and depression: Psychometric evidence and taxonomic implications [Press release] 
Craske, M. G., Meuret, A. E., Ritz, T., Treanor, M., Dour, H., \& Rosenfield, D. (2019). Positive affect treatment for depression and anxiety: A randomized clinical trial for a core feature of anhedonia. J Consult Clin Psychol, 87(5), 457-471. doi:10.1037/ccp0000396

Dagys, N., McGlinchey, E. L., Talbot, L. S., Kaplan, K. A., Dahl, R. E., \& Harvey, A. G. (2012). Double trouble? The effects of sleep deprivation and chronotype on adolescent affect. J Child Psychol Psychiatry, 53(6), 660-667. doi:10.1111/j.14697610.2011.02502.x

de Wild-Hartmann, J. A., Wichers, M., van Bemmel, A. L., Derom, C., Thiery, E., Jacobs, N., ... Simons, C. J. (2013a). Day-to-day associations between subjective sleep and affect in regard to future depressionin a female population-based sample. The British Journal of Psychiatry, 202(6), 407-412.

de Wild-Hartmann, J. A., Wichers, M., van Bemmel, A. L., Derom, C., Thiery, E., Jacobs, N., . . Simons, C. J. P. (2013b). Day-to-day associations between subjective sleep and affect in regard to future depressionin a female population-based sample. British Journal of Psychiatry, 202(6), 407-412. doi:10.1192/bjp.bp.112.123794

Edwards, C. J., Cella, M., Tarrier, N., \& Wykes, T. (2016). The optimisation of experience sampling protocols in people with schizophrenia. Psychiatry Res, 244, 289-293. doi:10.1016/j.psychres.2016.07.048

Geyer, E. C., Fua, K. C., Daniel, K. E., Chow, P. I., Bonelli, W., Huang, Y., . . Teachman, B. A. (2018). I Did OK, but Did I Like It? Using Ecological Momentary Assessment to Examine Perceptions of Social Interactions Associated With Severity of Social Anxiety and Depression. Behav Ther, 49(6), 866-880. doi:10.1016/j.beth.2018.07.009 
Gross, J. J., \& John, O. P. (2003). Individual differences in two emotion regulation processes: implications for affect, relationships, and well-being. J Pers Soc Psychol, 85(2), 348362. doi:10.1037/0022-3514.85.2.348

Hofmann, S. G., Sawyer, A. T., Fang, A., \& Asnaani, A. (2012). Emotion dysregulation model of mood and anxiety disorders. Depress Anxiety, 29(5), 409-416. doi:10.1002/da.21888

Hox, J. (2010). Multilevel Analysis. In G. A. Marcoluides (Ed.), (2nd ed.): Routledge: Taylor and Francis Group. .

Itani, O., Jike, M., Watanabe, N., \& Kaneita, Y. (2017). Short sleep duration and health outcomes: a systematic review, meta-analysis, and meta-regression. Sleep Med, 32, 246-256. doi:10.1016/j.sleep.2016.08.006

Jike, M., Itani, O., Watanabe, N., Buysse, D. J., \& Kaneita, Y. (2018). Long sleep duration and health outcomes: A systematic review, meta-analysis and meta-regression. Sleep Medicine Reviews, 39, 25-36. doi:https://doi.org/10.1016/j.smrv.2017.06.011

John, O. P., \& Gross, J. J. (2004). Healthy and unhealthy emotion regulation: personality processes, individual differences, and life span development. J Pers, 72(6), 13011333. doi:10.1111/j.1467-6494.2004.00298.x

Littlewood, D. L., Kyle, S. D., Carter, L.-A., Peters, S., Pratt, D., \& Gooding, P. (2019). Short sleep duration and poor sleep quality predict next-day suicidal ideation: an ecological momentary assessment study. Psychological medicine, 49(3), 403-411. doi:10.1017/S0033291718001009

McCulloch, C. E. (2003). Generalized Linear Mixed Models. NSF-CBMS Regional Conference Series in Probability and Statistics, 7, i-84. Retrieved from http://www.jstor.org/stable/4153190 
Morin, C. M., Belleville, G., Bélanger, L., \& Ivers, H. (2011). The Insomnia Severity Index: psychometric indicators to detect insomnia cases and evaluate treatment response. Sleep, 34(5), 601-608. doi:10.1093/sleep/34.5.601

Ong, A. D., Kim, S., Young, S., \& Steptoe, A. (2017). Positive affect and sleep: A systematic review. Sleep Medicine Reviews, 35, 21-32. doi:10.1016/j.smrv.2016.07.006

Palmer, C. A., \& Alfano, C. A. (2017). Sleep and emotion regulation: An organizing, integrative review. Sleep Medicine Reviews, 31, 6-16. doi:10.1016/j.smrv.2015.12.006

Persoskie, A., \& Ferrer, R. A. (2017). A Most Odd Ratio:: Interpreting and Describing Odds Ratios. Am J Prev Med, 52(2), 224-228. doi:10.1016/j.amepre.2016.07.030

Quoidbach, J., Berry, E. V., Hansenne, M., \& Mikolajczak, M. (2010). Positive emotion regulation and well-being: Comparing the impact of eight savoring and dampening strategies. Personality and Individual Differences, 49(5), 368-373. doi:https://doi.org/10.1016/j.paid.2010.03.048

Raes, F., Smets, J., Nelis, S., \& Schoofs, H. (2012). Dampening of positive affect prospectively predicts depressive symptoms in non-clinical samples. Cogn Emot, 26(1), 75-82. doi:10.1080/02699931.2011.555474

Reddy, R., Palmer, C. A., Jackson, C., Farris, S. G., \& Alfano, C. A. (2017). Impact of sleep restriction versus idealized sleep on emotional experience, reactivity and regulation in healthy adolescents. Journal of Sleep Research, 26(4), 516-525. doi:https://doi.org/10.1111/jsr.12484

Roenneberg, T., Kuehnle, T., Pramstaller, P. P., Ricken, J., Havel, M., Guth, A., \& Merrow, M. (2004). A marker for the end of adolescence. Current Biology, 14(24), R1038R1039. doi:10.1016/j.cub.2004.11.039 
Saksvik-Lehouillier, I., Saksvik, S. B., Dahlberg, J., Tanum, T. K., Ringen, H., Karlsen, H. R., .. . Olsen, A. (2020). Mild to moderate partial sleep deprivation is associated with increased impulsivity and decreased positive affect in young adults. Sleep, 43(10). doi:10.1093/sleep/zsaa078

Shen, L., van Schie, J., Ditchburn, G., Brook, L., \& Bei, B. (2018). Positive and Negative Emotions: Differential Associations with Sleep Duration and Quality in Adolescents. Journal of Youth and Adolescence, 47(12), 2584-2595. doi:10.1007/s10964-0180899-1

Short, N. A., Allan, N. P., \& Schmidt, N. B. (2017). Sleep disturbance as a predictor of affective functioning and symptom severity among individuals with PTSD: An ecological momentary assessment study. Behaviour Research and Therapy, 97, 146153. doi:https://doi.org/10.1016/j.brat.2017.07.014

Stone, L. B., Mennies, R. J., Waller, J. M., Ladouceur, C. D., Forbes, E. E., Ryan, N. D., .. . Silk, J. S. (2019). Help me Feel Better! Ecological Momentary Assessment of Anxious Youths' Emotion Regulation with Parents and Peers. Journal of abnormal child psychology, 47(2), 313-324. doi:10.1007/s10802-018-0454-2

Talbot, L. S., McGlinchey, E. L., Kaplan, K. A., Dahl, R. E., \& Harvey, A. G. (2010). Sleep deprivation in adolescents and adults: changes in affect. Emotion, 10(6), 831-841. doi:10.1037/a0020138

Tamm, S., Nilsonne, G., Schwarz, J., Golkar, A., Kecklund, G., Petrovic, P., . . Lekander, M. (2019). Sleep restriction caused impaired emotional regulation without detectable brain activation changes - a functional magnetic resonance imaging study. Royal Society Open Science, 6(3), 181704. doi:10.1098/rsos.181704 
Tavernier, R., \& Willoughby, T. (2015). A longitudinal examination of the bidirectional association between sleep problems and social ties at university: the mediating role of emotion regulation. $J$ Youth Adolesc, 44(2), 317-330. doi:10.1007/s10964-014-0107-x

The R Development Core Team. (2020). R: A language and environment for statistical computing. Vienna Austria. : R Foundation for Statistical Computing

van Zundert, R. M. P., van Roekel, E., Engels, R. C. M. E., \& Scholte, R. H. J. (2015). Reciprocal Associations Between Adolescents' Night-Time Sleep and Daytime Affect and the Role of Gender and Depressive Symptoms. Journal of Youth and Adolescence, 44(2), 556-569. doi:10.1007/s10964-013-0009-3

Verduyn, P., \& Brans, K. (2012). The relationship between extraversion, neuroticism and aspects of trait affect. Personality and Individual Differences, 52(6), 664-669. doi:https://doi.org/10.1016/j.paid.2011.12.017

Walker, M. P., \& van der Helm, E. (2009). Overnight therapy? The role of sleep in emotional brain processing. Psychol Bull, 135(5), 731-748. doi:10.1037/a0016570

Young, K. S., Sandman, C. F., \& Craske, M. G. (2019). Positive and Negative Emotion Regulation in Adolescence: Links to Anxiety and Depression. Brain Sci, 9(4). doi:10.3390/brainsci9040076 